\title{
Wireless RF Sensor Structure for Non-Contact Vital Sign Monitoring
}

\author{
Sang-Gyu $\mathrm{Kim}^{1} \cdot$ Gi-Ho Yun $^{2} \cdot$ Jong-Gwan Yook $^{1}$
}

\begin{abstract}
This paper describes a compact and novel wireless vital sign sensor at $2.4 \mathrm{GHz}$ that can detect heartbeat and respiration signals. The oscillator circuit incorporates a planar resonator, which functions as a series feedback element as well as a near-field radiator. The periodic movement of a human body during aerobic exercise could cause an input impedance variation of the radiator within near-field range. This variation results in a corresponding change in the oscillation frequency and this change has been utilized for the sensing of human vital signs. In addition, a surface acoustic wave (SAW) filter and power detector have been used to increase the system sensitivity and to transform the frequency variation into a voltage waveform. The experimental results show that the proposed sensor placed 20 $\mathrm{mm}$ away from a human body can detect the vital signs very accurately.
\end{abstract}

Key words: Planar Resonator, Series Feedback Oscillator, Vital Sign Sensor.

\section{Introduction}

The increasing demand for the detection of vital signs through wireless systems has focused considerable attention on the role of the RF (radio frequency) biosensor as a noncontact monitoring system for human healthcare and vital sign monitoring. This is especially true for cardiopulmonary monitoring of the sleep apnea syndrome and for patients whose severe burns preclude the attachment of electrodes or leads on the body [1]. Much recent research and many studies on wireless vital sign sensing have focused on the Doppler radar principle [2] $\sim$ [5]. Even though excellent performance has been achieved with wireless vital sign sensors based on Doppler radar theory, the existing Doppler radar approaches suffer from co-frequency interference. They are also incapable of detecting vital signs if more than one person is in the measurable range. Accordingly, Doppler radar has reached its limit of practical use in the medical area with conventional approaches. Related research and studies on the development of ECG using wet or dry electrodes have also been conducted [6] [7]. The use of these approaches is limited by the inconvenience and discomfort of wet adhesive electrodes and the need for direct contact with the body. As an alternative, non-contact capacitive electrodes [8] [10] have started to become much more commonplace, because these do not require an ohmic connection to the body and are insensitive to skin condition. However, this approach uti- lizes a static capacitance variation, which limits the measurement range to only a few mms.

This paper proposes a novel noncontact vital sign sensor topology for sensing of vital signs within a nearfield range at $2.4 \mathrm{GHz}$. The strategy is based on the fact that the oscillation frequency of an oscillator varies with the impedance variation of the planar resonator in the oscillator. In this work, the planar resonator has been used as a near-field radiator as well as a series feedback component. When the radiator is placed close to the body, the electromagnetic near-field produces additional capacitance between the body and the radiator. The additional capacitance consequently affects the impedance of the radiator when the body moves periodically due to aerobic exercise. The impedance variation of the radiator causes a corresponding change in the oscillation frequency of the oscillator, which is eventually utilized to detect human vital signs.

A surface acoustic wave (SAW) filter is also used to increase the sensitivity of the system. When the oscillator operates at the skirt frequency range of the SAW filter, a small amount deviation occurs in the oscillation frequency, which can be transformed to a large variation in the amplitude variation. A power detector is then used to detect the amplitude variation of the signal and convert this into a voltage waveform. As a result, the proposed detection mechanism can detect precise heartbeat and respiration signals in the near-field range.

\section{II . Operating Principle and System Configuration}

Manuscript received December 21, 2011 ; Revised January 31, 2012 ; Accepted February 10, 2012. (ID No. 20111221-040J)

${ }^{1}$ Degree in Electrical and Electronic Engineering, Yonsei University, Seoul, Korea.

${ }^{2}$ School of Information and Communication Engineering, Sungkyul University, Kyeonggi, Korea.

Corresponding Author : Jong-Gwan Yook (e-mail : jgyook@yonsei.ac.kr)

This is an Open-Access article distributed under the terms of the Creative Commons Attribution Non-Commercial License (http://creativecommons.org/licenses/ by-nc/3.0) which permits unrestricted non-commercial use, distribution, and reproduction in any medium, provided the original work is properly cited. 


\section{2-1 Operating Principle}

Fig. 1 shows the proposed vital sign sensor architecture. As shown in Fig. 1, the system consists of an oscillator, a SAW filter, a power detector, operational amplifier, and signal processing stage. As shown in Fig. 2(a), the frequency deviation of the oscillator due to subject movement is too small for detection and extraction of meaningful signals, especially for heartbeat information. A RF SAW filter is utilized at the oscillator output to enhance the sensitivity of the system, based on the property that the amplitude of the SAW filter output changes greatly in the skirt frequency band. As a result, a small frequency deviation in the oscillation frequency can be transformed into a large amplitude variation, as illustrated in Fig. 2(b). The RF power detector is then applied to the output of the SAW filter to produce a voltage waveform that represents respiration or heartbeat signals. In addition, the output of the RF power detector can be amplified by an operational amplifier. The output of the operational amplifier includes the desired vital signs as well as additional noise. The vital signs can be detected through appropriate digital signal processing and filtering.

In order to describe the operating principle, the frequency stability factor $S_{F}$ of oscillator and the relation between $S_{F}$ and phase variation of resonator should be defined. The definition of the $S_{F}$ is the change in phase divided by the normalized change in frequency from $\omega_{0}$ (i.e., $\Delta \omega / \omega_{0}$ ). Hence,

$$
S_{F}=\frac{\Delta \varphi}{\Delta \omega / \omega_{0}}=\left.\omega_{0} \cdot \frac{d \varphi}{d \omega}\right|_{\omega=\omega_{0}}
$$

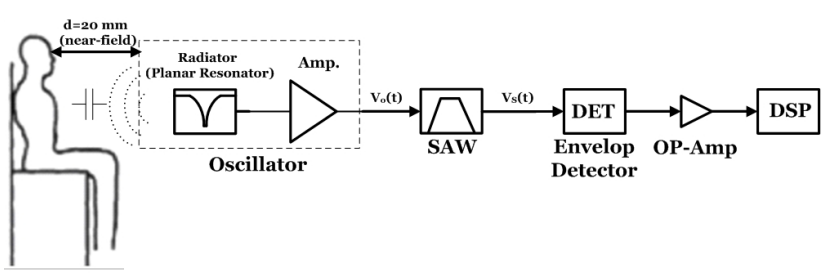

Fig. 1. Proposed vital sign sensor architecture.
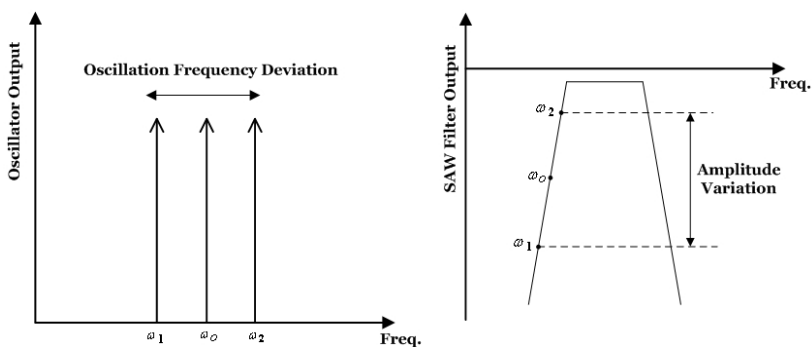

(a) Oscillator output spectrum (b) SAW filter output spectrum

Fig. 2. Output spectra.

$$
\Delta \omega=\frac{\omega_{0} \cdot \Delta \varphi}{S_{F}}
$$

The factor $S_{F}$ provides a qualitative way to compare the stability of oscillators. A large value of $S_{F}$ corresponds to a small frequency variation, as shown in (2). An oscillator is generally designed to have a higher $S_{F}$ value for this reason. However, a high $S_{F}$ value is not desired in the present case because a higher oscillation frequency variation is required for this application.

When the distance between the body and resonator is in the near-field range, the impedance of a planar resonator is changed due to vital signs such as respiration and pulse. If a living body is close to the planar resonator in a near-field range, additional capacitance between the body and resonator occurs, which alters the near field and, hence, change the resonator impedance. The impedance variation of the resonator can introduce or affect the location of the pole and zeros of the loop gain [11]. These variations in the poles and zeros introduce an additional phase shift $(\Delta \varphi)$, and the frequency of oscillation must shift an amount equivalent to $\Delta \omega$.

\section{2-2 Design of an Oscillator with a Planar Resonator as a Sensor}

Fig. 3(a) shows the configuration of a planar resonator. The resonator, which is determined by $\lambda_{g} / 2$, has a $16 \mathrm{~mm}$ radius. The overall size of the planar resonator realized on the FR-4board, including ground, is $100 \mathrm{~mm}$ by $100 \mathrm{~mm}$. The measured and simulated performances of the planar resonator are shown in Fig. 3 (b). The simulation is performed with a 3-dimensional full-wave electromagnetic solver and shows excellent agreement with the measurement. Resonance occurs at $2.38 \mathrm{GHz}$ with $3 \mathrm{~dB}$ bandwidth of $60 \mathrm{MHz}$. Proper re-
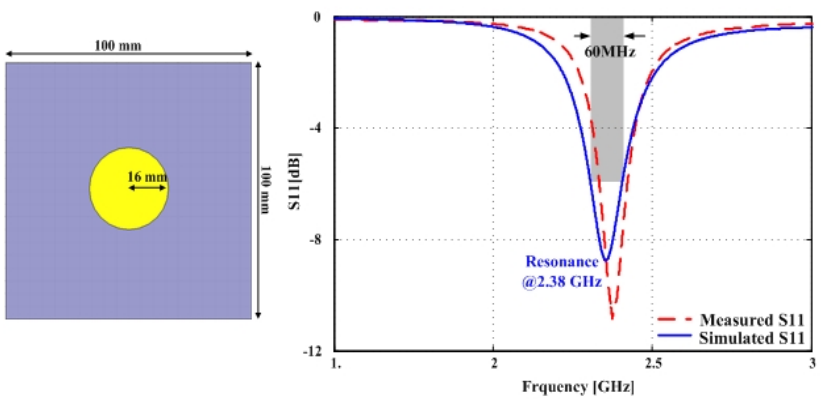

(a) Dimension and shape of the planar resonator

(b) Measured and simulated results of the resonator

Fig. 3. Shape of planar resonator and measurement results. 


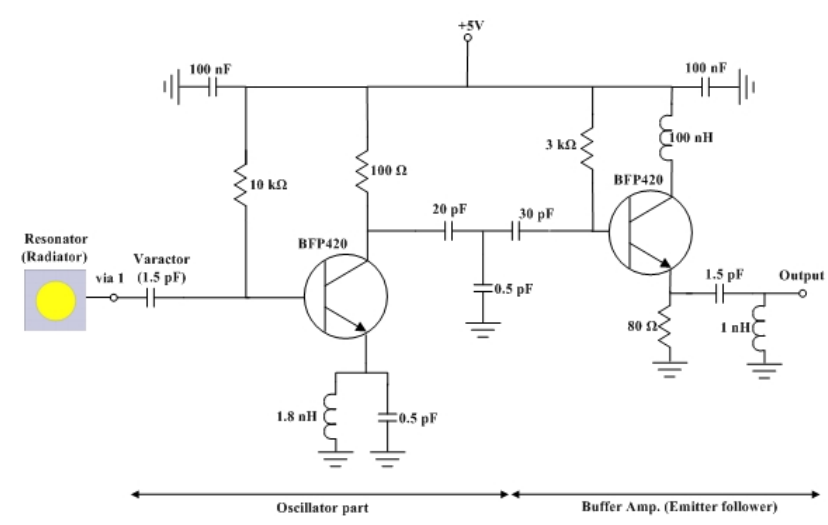

Fig. 4. Schematic diagram of the oscillator with a planar resonator and buffer amplifier.

active impedance control around bipolar transistor achieves an oscillation condition at the resonance frequency of the planar resonator [12] [14]. Fig. 4 shows a schematic diagram of the $2.4 \mathrm{GHz}$ amplifier and the cascade type resonator [15] [17] combined with an emitter follower type buffer amplifier. The resonator is placed at the base circuit of the transistor, and varactor between them adjusts the oscillation frequency. Close attention also has to be paid when the SAW filter is directly connected to the oscillator, since the oscillation condition can be changed by rapid variation in the impedance at the skirt frequency range of the SAW filter. For this reason, the emitter follower type buffer amplifier is used to prevent a strong mutual interaction between the oscillator and filter.

Two PCBs of the same size $(100 \mathrm{~mm}$ by $100 \mathrm{~mm}$ ) were fabricated, one for the circuit part and the other for the planar resonator. Both PCBs are connected through a via hole, as shown in Fig. 5. Consequently, as shown in Fig. 6, the measured oscillator output power at 2.375 $\mathrm{GHz}$, including the output of the emitter follower buffer

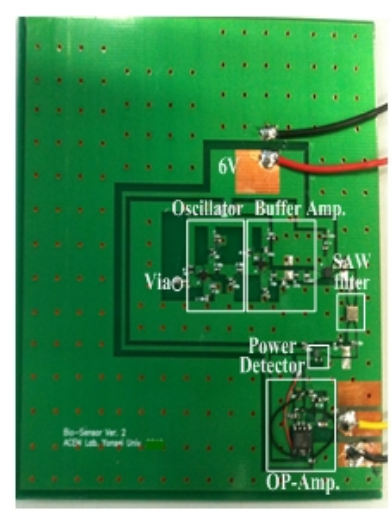

(a) Top (circuit part)

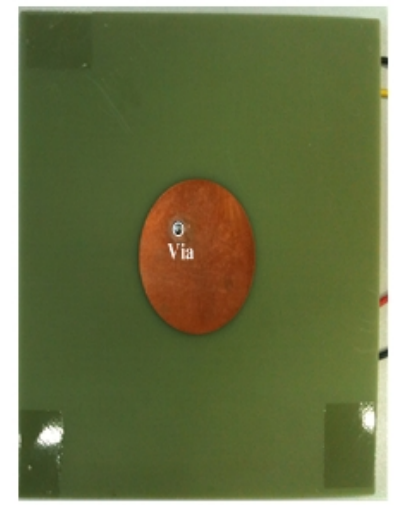

(b) Bottom (resonator part)
Fig. 5. Fabricated PCBs.

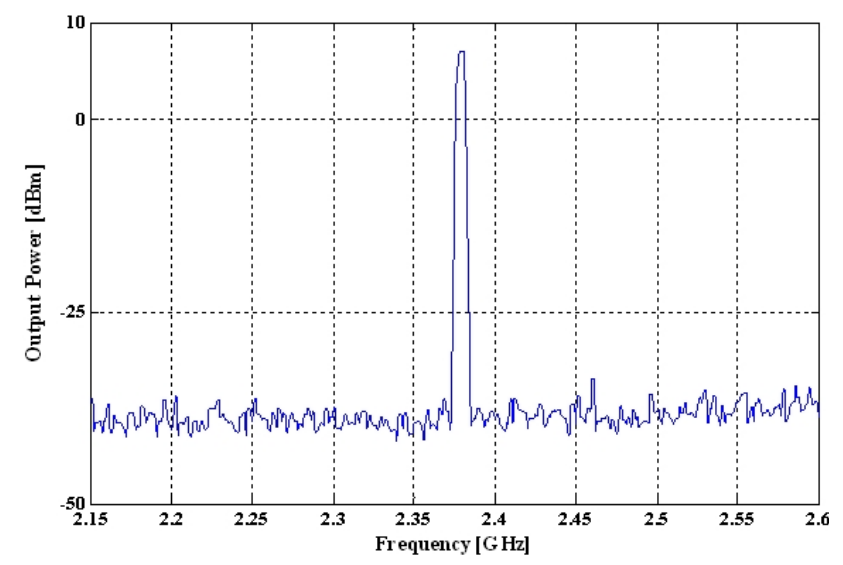

Fig. 6. Measured output power spectrum.

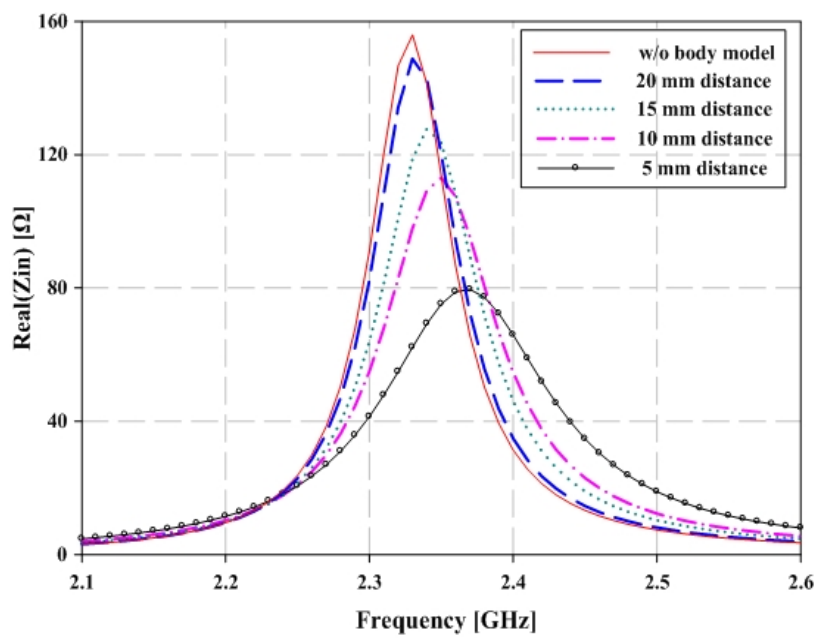

(a) Real part

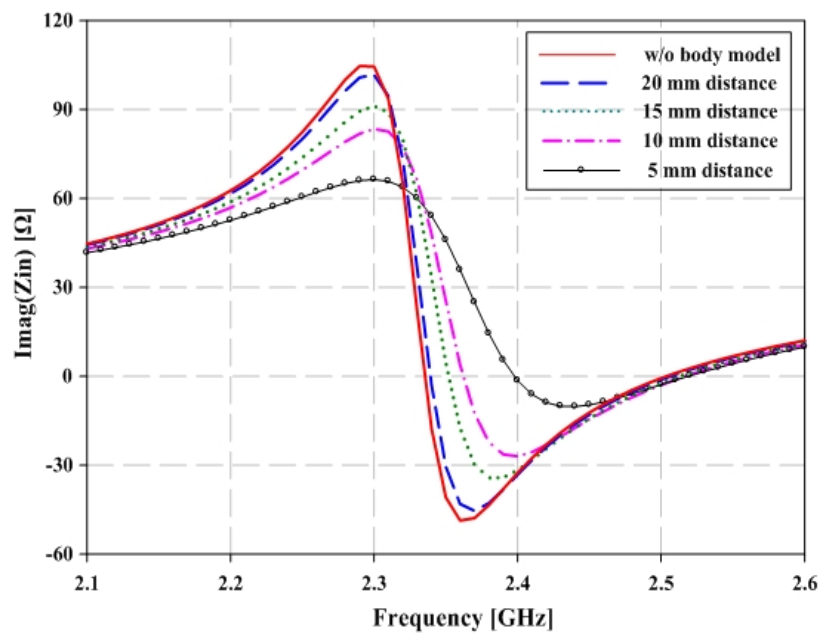

(b) Imaginary part

Fig. 7. Input impedance variation of resonator according to the distance between resonator and body.

amplifier, is $4 \mathrm{dBm}$.

When the resonator is brought close to the body object, the periodic movement alters the near-field and, 
hence, changes the resonator input impedance $\left(Z_{i n}\right)$. As mentioned previously, the oscillation frequency eventually varies with the physical movement of the subject. The near-field radiation characteristics of the planar resonator are investigated with a three dimensional electromagnetic field simulation of the planar resonator including a human body model and the input impedance variation of the resonator near the body object is calculated.

For effective and accurate modeling, the resonator is placed in front of a simplified human body model $\left(\right.$ a $30 \times 30 \times 30 \mathrm{~cm}^{3}$ cube with a permittivity value of 52.8 and a conductivity value of $1.7 \mathrm{~S} / \mathrm{m}$ ) [18]. The distance between the simplified body model and the resonator is varied from $1 \mathrm{~mm}$ to $40 \mathrm{~mm}$ with $1 \mathrm{~mm}$ intervals. Figs. 7(a) and (b) show the variance of the real and imaginary parts of the resonator input impedance as a function of the distance between the resonator and the body object. The calculated $S$-parameter and the input impedance variation are taken into account in the oscillator circuit simulation to verify the deviation in the oscillation frequency.

Fig. 8 summarizes the average rate of change for oscillation frequency deviation and amplitude variation at SAW filter output power within $10 \mathrm{~mm}$ and over 10 $\mathrm{mm}$ distance. When the distance between the resonator and the body model is smaller than $10 \mathrm{~mm}$, the input impedance of the resonator displays a quite noticeable variation when compared to the freestanding case. An average rate of change for this oscillation frequency deviation is $1.66 \mathrm{MHz} / \mathrm{mm}$. When the distance is greater than $10 \mathrm{~mm}$, the average rate of change for the oscillation frequency deviation is $0.33 \mathrm{MHz} / \mathrm{mm}$. This means that the body object has a negligible effect on the input

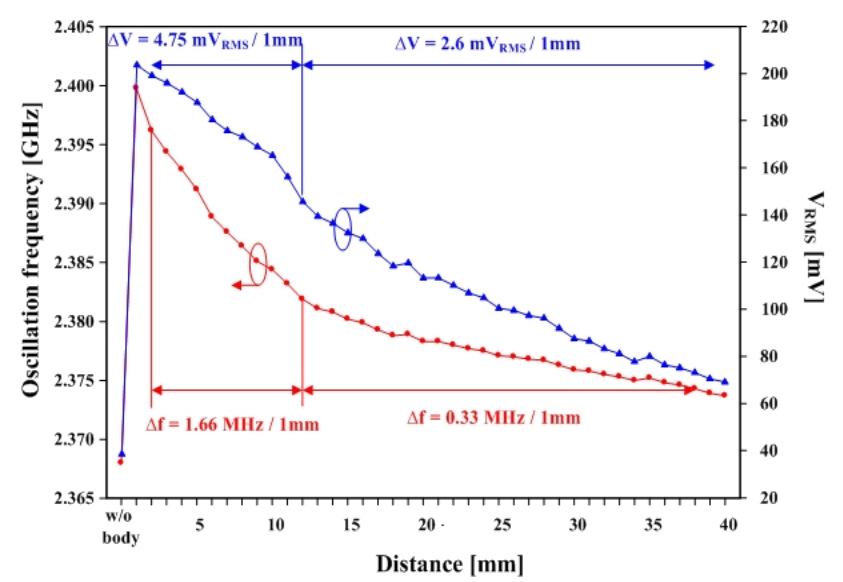

Fig. 8. Simulated the average rate of change for oscillation frequency deviation and amplitude variation at the SAW filter output in relation to the distance between the resonator and body model.
Table 1. Oscillation frequency and SAW filter output power in relation to the distance between the body and the resonator.

\begin{tabular}{|c|c|c|c|c|}
\hline \multirow{2}{*}{$\begin{array}{c}\mathrm{D} \\
{[\mathrm{mm}]}\end{array}$} & \multirow{2}{*}{$\begin{array}{l}\text { Simulated } \\
\text { oscillation } \\
\text { freq. [GHz] }\end{array}$} & \multirow{2}{*}{$\begin{array}{c}\text { SAW output } \\
\text { voltage } \\
{[\mathrm{mV}]}\end{array}$} & \multicolumn{2}{|c|}{$\begin{array}{l}\text { Average rate of } \\
\text { change for }\end{array}$} \\
\hline & & & $\begin{array}{c}\text { Frequency } \\
\text { deviation }\end{array}$ & $\begin{array}{c}\text { Amplitude } \\
\text { variation }\end{array}$ \\
\hline 0 & 2.37 & 37 & \multirow{11}{*}{$\begin{array}{c}1.66 \\
\mathrm{MHz} / \mathrm{mm}\end{array}$} & \multirow{11}{*}{$\begin{array}{c}4.7 \\
\mathrm{mV} / \mathrm{mm}\end{array}$} \\
\hline 1 & 2.3998 & 206 & & \\
\hline 2 & 2.3962 & 201 & & \\
\hline 3 & 2.3944 & 197 & & \\
\hline 4 & 2.3929 & 194 & & \\
\hline 5 & 2.3912 & 186 & & \\
\hline 6 & 2.3889 & 181 & & \\
\hline 7 & 2.3876 & 178 & & \\
\hline 8 & 2.3864 & 177.2 & & \\
\hline 9 & 2.3851 & 170 & & \\
\hline 10 & 2.3844 & 164 & & \\
\hline 11 & 2.3832 & 157 & \multirow{10}{*}{$\begin{array}{c}0.33 \\
\mathrm{MHz} / \mathrm{mm}\end{array}$} & \multirow{10}{*}{$\begin{array}{c}2.6 \\
\mathrm{mV} / \mathrm{mm}\end{array}$} \\
\hline 12 & 2.3819 & 147 & & \\
\hline 13 & 2.3811 & 140 & & \\
\hline 14 & 2.3808 & 138 & & \\
\hline 15 & 2.3802 & 135 & & \\
\hline 16 & 2.3799 & 130 & & \\
\hline 17 & 2.3793 & 123 & & \\
\hline 18 & 2.3788 & 119 & & \\
\hline 19 & 2.3789 & 121 & & \\
\hline 20 & 2.3783 & 117 & & \\
\hline
\end{tabular}

impedance of the resonator. This degree of variation is also considered to be very small for a direct detection scheme for vital signs. The implementation of detection system for small frequency deviations, such a few hundred $\mathrm{kHz}$, is very difficult and complicated at $2.4 \mathrm{GHz}$. Traditionally, a frequency synthesizer was used to transform the frequency deviation into an amplitude variation [19], which causes a system complexity.

The output of proposed vital sign sensor should also be represented by a time domain signal. However, the average rate of change for the magnitude variation in the skirt range (from $2.35 \mathrm{GHz}$ to $2.395 \mathrm{GHz}$ ) of a SAW filter is about $1 \mathrm{~dB} / \mathrm{MHz}$. Therefore, the average rate of change for amplitude variations of 1.66 and 0.33 $\mathrm{dB} / \mathrm{mm}$ can be achieved by a SAW filter within $10 \mathrm{~mm}$ and over $10 \mathrm{~mm}$ distance, respectively. For these reasons, the use of the SAW filter is the simplest and most effective method for transforming the small frequency variation into a large amplitude variation and the maximum distance of the proposed vital sensor is estimated 


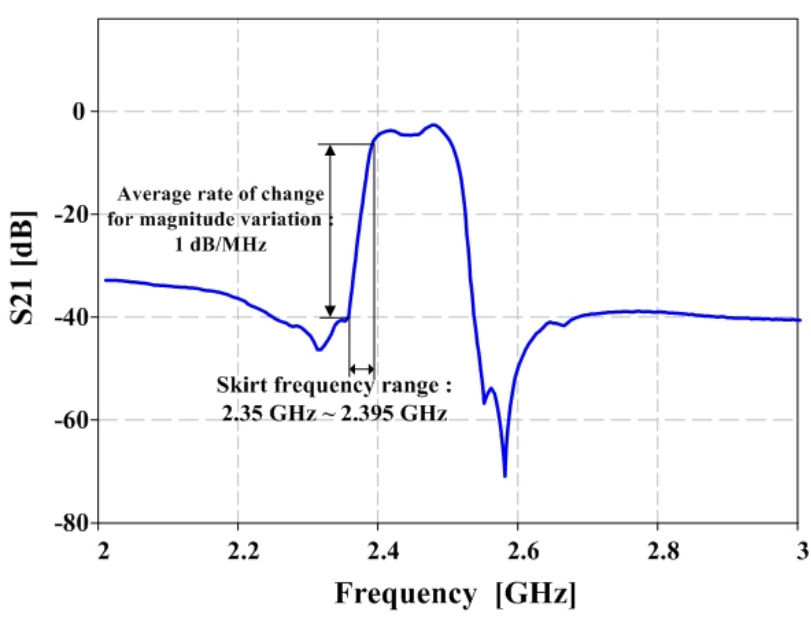

Fig. 9. Measurement results of SAW filter.

about $10 \mathrm{~mm}$. A SA2441AM SAW filter from SAWNICS is used and the measured performance of the SAW filter is shown in Fig. 9. The designed oscillator operates at $2.375 \mathrm{GHz}$ in the skirt frequency range $(2.35$ $\mathrm{GHz}$ to $2.395 \mathrm{GHz}$ ) of the SAW filter. Thus, it enables small frequency deviations to be transformed into a large amplitude variations at the output voltage.

The signal is then detected by RF power detector using an AD8312 RF detector (Analog Devices Inc.). It has a frequency response from $50 \mathrm{MHz}$ to $3.5 \mathrm{GHz}$ and detection power level from $-45 \mathrm{dBm}$ to $0 \mathrm{dBm}$. The amplitude variation is found to be $4.75 \mathrm{mV}_{\mathrm{RMS}} / \mathrm{mm}$ within $10 \mathrm{~mm}$ distance and $2.6 \mathrm{mV}_{\mathrm{RMS}} / \mathrm{mm}$ within 20 $\mathrm{mm}$ distance. Thus, the proposed vital sign sensor structure is able to detect the amplitude variation due to vital exercise with a straightforward power detection method.

\section{Measurement Results}

Our proposed direct detection vital sign sensor system has been designed and fabricated on the basis of the vital sign sensing mechanism mentioned in Section II. To verify the performance of the developed vital sign sensor, a reference heartbeat signal by finger-pressure heartbeat sensor (UFI-1010) is measured simultaneously, as illustrated in Fig. 10. The sensor is fixed on a tripod 20 $\mathrm{mm}$ from the body and the measurements of the two signals are acquired and digitized by an oscilloscope (Tektronix TDS2014). The human subject is seated on a chair and breathing, and the vital sign sensor is placed at the center of the subject's chest. The data digitized by the oscilloscope are transferred to a computer through a GPIB interface. In the first step of digital signal processing, the incoming time domain signal, which includes a fair amount of noise, is transformed into a frequency domain signal by a discrete Fourier Transform (DFT) to

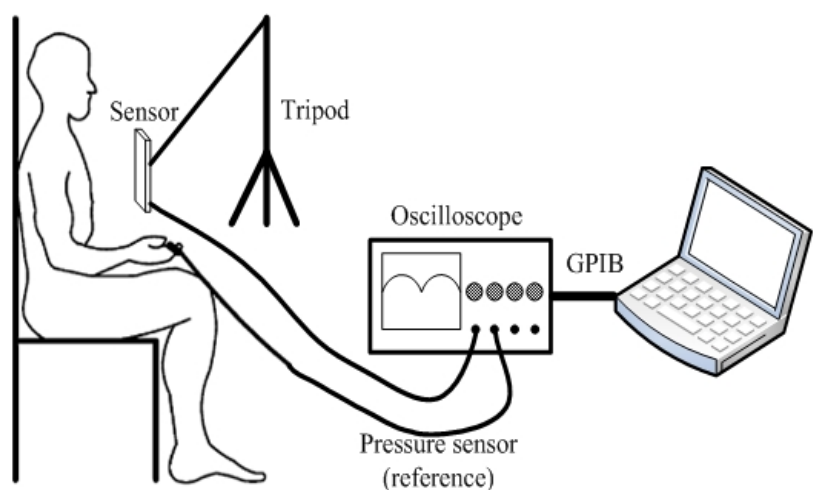

Fig. 10. Measurement setup for pulse and respiration detection.

confirm whether the desired signal exists in the appropriate frequency range. The noise components that accompany the vital signal can be removed by a frequency domain filtering method (heartbeat: $0.8 \mathrm{~Hz}$ to $5 \mathrm{~Hz}$ band-pass filtering). After filtering, the respiration and heartbeat signals are transformed into a time domain signal by an Inverse discrete Fourier Transform (IDFT) and displayed as a vital sign waveform. Fig. 11(a) shows a digitized time domain waveform from the oscilloscope; Fig. 11(b) shows the frequency domain waveform before any filtering. The time domain waveform in Fig. 11(a) includes the heartbeat and respiration signals. Because the respiration signal has a larger magnitude than the heartbeat signal, it can be easily extracted.

Fig. 11(b) shows the frequency domain signal output of the proposed sensor. The DFT result clearly shows that the output signals of the proposed vital sensor include heartbeat (at $0.55 \mathrm{~Hz}$ ) as well as respiration (at $1.15 \mathrm{~Hz}$ ) components. The heartbeat information can be

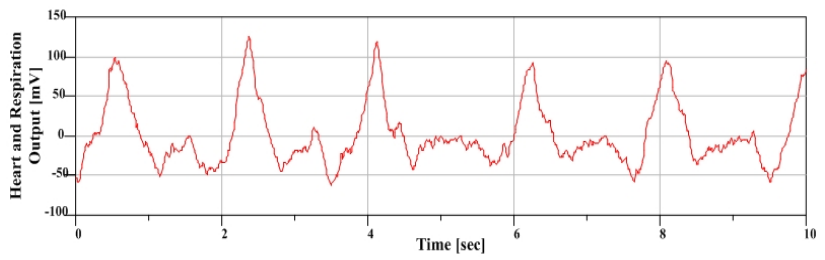

(a) Output time domain waveform

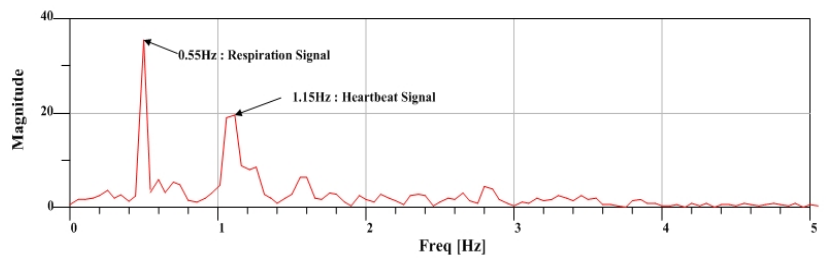

(b) DFT results of time domain waveform of the proposed sensor

Fig. 11. Measured output signal of the vital sensor at a distance of $20 \mathrm{~mm}$. 


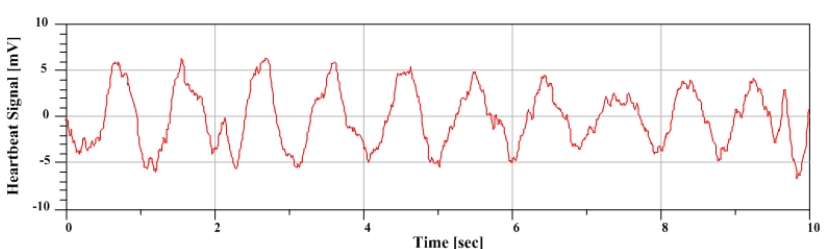

(a) Result of the waveform after filtering results from the proposed sensor

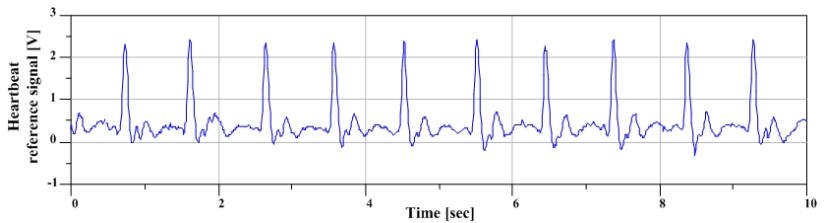

(b) Result of the time domain waveform from the finger sensor

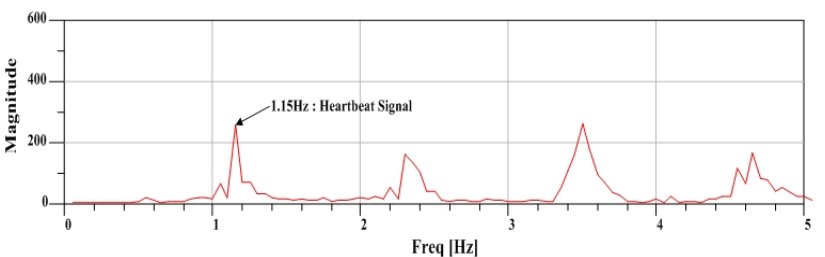

(c) DFT result of the time domain waveform from the finger sensor

Fig. 12. Heartbeat signal detection at distance of $20 \mathrm{~mm}$.

separated by applying a simple digital filter with a band pass filter with a pass-band of $0.8 \mathrm{~Hz}$ to $5 \mathrm{~Hz}$. Fig. 12 (a) shows the time domain waveform of the heartbeat signal after band-pass filtering and inverse Fourier transformation. The accuracy of the detected heartbeat signal is verified by simultaneously measuring heartbeat with a finger-pressure heartbeat sensor (UFI-1010), as shown in Fig. 12(b). Fig. 12(c) shows the frequency domain data obtained from the finger sensor. The spectra in Figs. 11 (b) and 12(c) both show a strong peak at an identical frequency of $1.15 \mathrm{~Hz}$. The measured respiration rate is 33 beats $/ \mathrm{min}$ and the heartbeat rate is 69 beats $/ \mathrm{min}$.

\section{Conclusion}

This paper presents a new architecture for a wireless vital sign sensor system. The proposed vital sign sensor utilizes the frequency deviation of an oscillator induced by the impedance variation of planar resonator in nearfield range. The planar resonator functions as a sensor as well as a positive feedback element of the oscillator. In addition, the skirt characteristics of a SAW filter are cleverly exploited to improve the sensitivity of the system and a power detector is used to convert the oscillation frequency deviation into a time domain voltage waveform. A discrete Fourier transform procedure is used in the DSP to extract the frequency component of vital signs. The proposed vital sign sensor can detect a respiration signal as well as a heartbeat signal in the near-field range. Our measurements confirm that a longer measurement range is achieved than for electrode method sensors that detect static capacitance variation. The proposed method is more practical than Doppler radar methods due to the lack of restrictions on the measurement environment and it has potential use in medical monitoring equipment, the automobile industry, and any portable/wearable applications.

This research was supported by the MKE(The Ministry of Knowledge Economy), Korea, under the ITRC(Information Technology Research Center) support program supervised by the NIPA(National IT Industry Promotion Agency)" (NIPA-2011-(C10901121-0005))

\section{References}

[1] V. M. Lubecke, Boric-Lubecke, A. Host-Madsen, and A. E. Fathy, "Through-the-wall radar life detection and monitoring," IEEE MTT-S Int. Microwave Symposium Digest, pp. 769-772, May 2007.

[2] S. G. Kim, H. Kim, Y. Lee, I. S. Kho, and J. G. Yook, "5.8 GHz vital signal sensing Doppler radar using isolation-improved branch-line coupler," Radar Conference $3^{\text {rd }}$, EuMW, Sep. 2006.

[3] Y. Yan, C. Li, and J. Lin, "Effect of I/Q mismatch on measurement of periodic movement using a Doppler radar sensor," Proc. IEEE Radio and Wireless Symp., pp. 196-199, Jan. 2010.

[4] Y. Xiao, J. Lin, O. Boric-Lubecke, and V. M. Lubecke, "Frequency tuning technique for remote detection of heartbeat and respiration using low-power double-sideband transmission in a-band," IEEE $T r$ ans. Microw. Theory Tech., vol. 54, no. 5, pp. 20232032, May 2006.

[5] A. D. Droitcour, O. Boric-Lubecke, V. M. Lubecke, J. Lin, and G. T. A. Kovac, "0.25 um CMOS and BiCMOS single chip direct conversion Doppler radars for remote sensing of vital signs," IEEE Int. Solid State Circuits Conf. Dig., pp. 348-349, Feb. 2002.

[6] T. J. Sullivan, S. R. Deiss, T. P. Jung, and G. Cauwenberghs, "A brain machine interface using drycontact, low noise EEG sensors," IEEE International Symp., pp. 1986-1989, 2008.

[7] Alfredo Lopez, P. C. Richardson, "Capacitive electrocardiographic and bioelectrodes," IEEE Trans. on Biomedical Engineering., vol. 1, pp. 96-99, Jan. 1969. 
[8] T. J. Sullivan, S. R. Deiss, and G. Cauwenberghs, "A low-noise, non-contact EEG/ECG sensor," in Biomedical Circuit and Systems Conf., pp. 154-157, 2007.

[9] C. Park, P. H. Chou, Y. Bai, R. Matthews, and A. Hibbs, "An ultra-wearable, wireless, low power ECG monitoring system," in Biomedical Circuits and Systems Conf., pp. 241-244, Nov. 2006.

[10] A. Ueno, Y. Akabanem, T. Kato, H. Hoshino, S. Kataoka, and Y. Ishiyama, "Capacitive sensing of electrocardiographic potential through cloth from the dorsal surface of the body in a supine position: A preliminary study," IEEE Trans. on Biomedical Engineering, vol. 54, pp. 759-766, Apr. 2007.

[11] Jeremy Everard, Fundamentals of RF Circuit Design with Low Noise Oscillators, John Wiley \& Sons, Ltd., 2001.

[12] Randall W. Rhea, Oscillator Design and Computer Simulation, $2^{\text {nd }}$ edition, Novel Publishing Corporation, 1995.

[13] Jeremy Everard, Fundamentals of RF Circuit Design with Low Noise Oscillators, John Wiley \& Sons, Ltd., 2001.

[14] F. Giannini, G. Leuzzi, Nonlinear Microwave Cir-

\section{Sang-Gyu Kim}

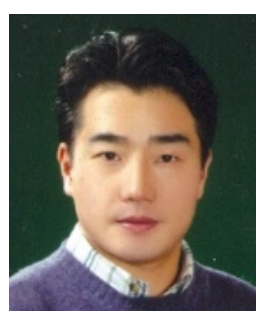

was born in Seoul, Korea. He received the B.S and M.S degrees in electrical and electronic engineering from KyungwonUniversity, Korea in 2001, 2003, respectively, and is currently pursuing the $\mathrm{Ph} . \mathrm{D}$. degree in electrical and electronic engineering at the Yonsei University, Seoul, Korea. From December 2005 to December 2008, he worked in Analog Devices Inc., Korea, as a Packaging Design Engineer. His research interests are theoretical/numerical electromagnetic modeling, characterization of microwave circuit, analysis and optimization of high-speed interconnection, including electromagnetic interference (EMI)/ electromagnetic compatibility (EMC), and remote wireless vital signal monitoring sensors. cuit Design, John Wiley \& Sons, Ltd., 2004.

[15] A. A. Behagi, S. D. Turner, "A non-linear CAD model for the regenerative dielectric resonator oscillator," IEEE International Frequency Control Symposium, 1995.

[16] A. Takaoka, K. Ura, "Noise analysis of nonlinear feedback oscillator with AM-PM conversion coefficient," IEEE Trans. Microwave Theory and Tech., vol. 28, no. 6. Jun. 1980.

[17] M. Regis, O. Llopis, and J. Graffeuil, "Nonlinear modeling and design of bipolar transistors ultra low phase-noise dielectric-resonator oscillators," IEEE $\mathrm{Tr}$ ans. Microwave Theory and Tech., vol. 46, no. 10, Oct. 1998

[18] C. Gabriel, S. Gabriel, and E. Corthout, "The dielectric properties of biological tissues: I. Literature survey," Phy. Med. Biol., 41, 2231, 1996.

[19] M. Jaeger, M. Mueller, D. Wettach, T. Oezkan, J. Motsch, T. Schauer, R. Jaeger, and A. Bolz, "Firstaid sensor system : New methods for single-point detection and analysis of vital parameters such as pulse and respiration," IEEE Engineering in Medicine and Biology Society, Aug. 2007.

\section{Gi-Ho Yun}

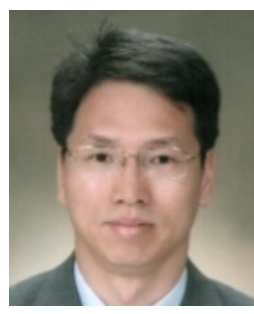

was born in Jeonju-Si, Korea. He received the B.S., M.S. and Ph.D. degrees in electronics engineering from Yonsei University, Seoul, Korea, in 1984, 1986 and 1999, respectively. From 1985 to 1997, He worked at Samsung Electronics and Samsung Electro-Mechanics. He also served at Honam University from 1997 to 2008. He is currently an assistant professor in the School of Information and Communication Engineering, Sungkyul University, Kyeonggi-Do, Korea. He is currently working with Advanced Computational Electromagnetic Lab. of Yonsei University. His research interests include radio frequency (RF) circuits and patch antenna, and biosensors. 


\section{Jong-Gwan Yook}

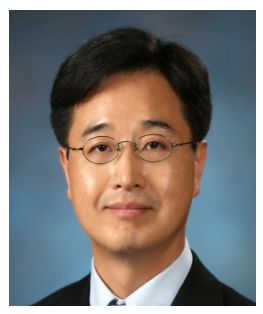

(S'89 M'97) was born in Seoul, Korea.

He received the B.S. and M.S. degrees in electronics engineering from Yonsei University, Seoul, Korea, in 1987 and 1989, respectively, and the Ph.D. degree from The University of Michigan, Ann Arbor, MI, in 1996. He is currently a Professor with the School of Electrical and Electronic Engineering, Yonsei University. His main research interests are in the areas of theoretical/numerical electromagnetic modeling and characterization of microwave/millimeter-wave circuits and components, design of radio frequency integrated circuits (RFIC) and monolithic microwave integrated-circuit (MMIC), and analysis and optimization of high-frequency high-speed interconnects, including signal/power integrity (EMI/EMC), based on frequency as well as time-domain full-wave methods. Recently, his research team is developing various biosensors, such as carbon-nano-tube RF biosensor for nanometer size antigen-antibody detection as well as remote wireless vital signal monitoring sensors. 